\title{
A Qualitative Study to Explore Adolescent Girls Belief on Menstruation and Health Seeking Behavior
}

\author{
Karuna Bhattarai ${ }^{1, ~ *, ~ R a j e n d r a ~ K a r k e e ~}{ }^{1}$, Anup Ghimire ${ }^{1}$, Prajjwal Pyakurel ${ }^{1}$, \\ Purna Prasad Sharma ${ }^{2}$, Tara Ballav Adhikari ${ }^{3}$, Rama Shrestha ${ }^{4}$ \\ ${ }^{1}$ School of Public Health and Community Medicine, B. P. Koirala Institute of Health Sciences, Dharan, Nepal \\ ${ }^{2}$ Tikapur Hospital, Tikapur Kailali, Nepal \\ ${ }^{3}$ Nepal Health Frontiers, Maharajgunj, Kathmandu, Nepal \\ ${ }^{4}$ National Academy of Medical Sciences, Kathmandu, Nepal
}

Email address:

bhattaraikaruna3@gmail.com (K. Bhattarai)

${ }^{*}$ Corresponding author

\section{To cite this article:}

Karuna Bhattarai, Rajendra Karkee, Anup Ghimire, Prajjwal Pyakurel, Purna Prasad Sharma, Tara Ballav Adhikari, Rama Shrestha. A Qualitative Study to Explore Adolescent Girls Belief on Menstruation and Health Seeking Behavior. Biomedical Sciences.

Vol. 6, No. 2, 2020, pp. 31-37. doi: 10.11648/j.bs.20200602.13

Received: April 25, 2020; Accepted: May 18, 2020; Published: June 16, 2020

\begin{abstract}
Background: To study issues related to menstruation and health seeking practices to address the importance of the reproductive health of adolescent girls. Methods: A qualitative study was conducted in Surkhet district, Province 6 of Western Nepal. Information was collected from four focus group discussions (FGDs). Two FGDs were conducted among adolescent girls and remaining two from mothers having adolescent daughters. Results: FGDs show that most of the adolescent girls were shy and anxious during menarche. Some of them could not tell their mother too. Majority of them restricted to do some activities and consume some food items during menstruation. Yet, the practice of visiting"dhami jhakri" and then only going to the health facility after the problem has become severe. Although menstruation is normal physiology, in Nepal menstruating woman is categorized as "impure", "polluted" and victimized in the form of "Chhaupadi Pratha". The misconception that "females touching others during their menstruation brings God's curse to their home and families" is still rampant in Western Nepal. Conclusion: Concerned authorities should conduct health promotion activities to address menstrual hygiene and other traditional practices among adolescent girls in the Western Nepal. The findings of this study will be useful for healthcare professional and administrator to improve the reproductive health status of the adolescent.
\end{abstract}

Keywords: Adolescent Girls, Menstrual Practice, Health Seeking Behavior, Nepal

\section{Introduction}

Adolescence is also referred as a phase of rapid physical and cognitive growth. World Health Organization (WHO) defines people of age group between 10 to19 years as adolescence. Globally, there are 1.2 billion adolescences. In Nepal adolescents comprise 24 percent of the total population [1]." In this transition from childhood to adulthood they become vulnerable to a number of problems such as psychosocial problems, general and reproductive health problems, sexuality related problems that requires special protection and attention [2]." All societies need to recognize adolescent development, which drives the changes in the disease burden between childhoods to adulthood [3]."

The onset of menstruation is an important physiological change in adolescent girls. It is defined as the initiation of uterine bleeding and is often marked as a potential entry into sexual relationships and reproduction. First menstruation is often a traumatic and very negative experience for young girls in most parts of the developing countries. Several traditional beliefs, misconceptions and practices are linked to the issue of menstruation, which makes girls vulnerable to depression and stress as well as reproductive problems [4]."

Although menstruation is normal physiology, in Nepal menstruating woman is categorized as "impure", "polluted" 
and victimized in the form of "Chhaupadi Pratha". They are treated inhumanely and segregated from usual life and forced to live in an unhygienic place known as "Chhaupadi Gotha" and follow lack of nutritious food. This practice not only deteriorates women's health, but also violates their basic rights and individual freedom and also puts them at other risk [5]." There are no any good effects of traditional menstrual practices except one i.e. menstruating girls are not allowed to do household work at that time and they get an opportunity to take rest.

Forced to remain outdoors during the entire length of their menstrual cycle because of fear of bringing down the Goddess's curse on their home and family, there are other restrictions such as not touching a man, not touching a cow and not touching any fruit trees. If they do, it is believed that the tree will stop producing fruit, the cow will stop producing milk and the man will fall ill. Prohibited from bathing during their cycle, women are forced to live in the unsanitary condition of a hut especially made for them. These are the most common cultural practices and misconceptions, which are still prevalent in our society and directly or indirectly influence health seeking behavior for menstrual problems $[6,7]$." Hence, this study aims to explore the issues related to menstruation and health seeking practices to address the importance of the reproductive health of adolescent girls.

\section{Methods}

\subsection{Study Area and Population}

Province no. 6 and 7 (Mid and Far Western Region) are at high risk of reproductive health problems. Different news and reports show higher menstrual related issues in these two regions. ${ }^{7}$ Surkhet district was hence chosen for this purpose. Even though Surkhet has high literacy rate $(96.26 \%)$, health seeking behavior among adolescents is not satisfactory, so, it is important to know the factors for hindering health seeking behavior. In some parts of Surkhet district, "Chaupadi Pratha" is still prevailing due to which most of the adolescent girls experience various reproductive health problems and also become victims of snake bite, rape and other problems.

Adolescent girls from Public schools of Rural and Urban Municipality were recruited for focus group discussion. Similarly, mother participants were chosen from Rural and urban community area for this study purpose.

\subsection{Recruitment and Participants}

Focus Group Discussion (FGD) was carried out among adolescent girls and mothers. Total six secondary level schools were chosen randomly among them three secondary level schools were selected from Rural Municipality and remaining three schools from Urban Municipality. Three adolescent girls were selected from each of the sampled secondary level public school of Rural Municipality for one
FGD (9 adolescent girls in each group) and the same procedure was applied in Urban Municipality for another FGD (Total nine students in each FGD). With the help of FCHVs, other two FGDs were conducted with mothers (9 mothers in each group): one in rural area and other in urban area.

Participants were explained about the study objectives, and both verbal and written consents were taken before starting the discussions.

\subsection{Focus Group Approach}

This is an exploratory qualitative study conducted in Surkhet district, Province 6 of Western Nepal. Information was collected from four Focus Group Discussions (FGDs). Two FGDs were conducted among adolescent girls and remaining two from mothers having adolescent daughters.

FGD guideline was used to conduct FGD with adolescent girls and mothers. The guideline included: Knowledge and feeling/experience about menstruation at the time of menarche Reproductive health problems and menstrual problem in community, Belief on menstruation and Health seeking practices at household level. FGD was conducted in Nepali language by using FGD guideline. Each FGD lasted for about one hour. The discussions were transcribed in Nepali language and translated into English. The transcripts were then reviewed by guides and coguides for accuracy.

\subsection{Ethical Clearance}

The purpose of the study and procedures was explained. Participants were informed that about voluntary participation and given information was used only for the research purpose. Written informed consent from respondent's parent and verbal consent from respondent was gained before starting the FGDs. They were assured that their responses would be treated in confidence and anonymity through the use of strict coding measures. All information was kept confidential and consent forms were number coded for identification.

Ethical Clearance was obtained from the Institutional Review Committee, BPKIHS, Dharan, Nepal. (Institutional Review Committee Ref. No. 277/074/075-IRC).

\subsection{Data Analysis}

Information from the Focus Group Discussion were transcribed and recorded. Information was taken until saturation point. Thematic analysis was done by using different theme which was emerged from the discussion.

The English language transcripts were read several times for familiarity with the raw data and to frame the information by using the key themes which emerged. The framework was divided further into subthemes. The narratives were then recoded where relevant, under these subthemes. Based on different them info graph was made which make easier to display the major results of FGD. 


\section{Results}

Table 1. General Description of Focus Group Discussion.

\begin{tabular}{|c|c|c|c|c|}
\hline FGD & Category & & Number of participants (n) & Venue \\
\hline \multirow{3}{*}{ FGD1 } & \multirow{2}{*}{ Age Group } & Early adolescent (14-15 years) & 6 & \multirow{3}{*}{ Birendranagar Municipality, Surkhet } \\
\hline & & Late adolescent ( $16-19$ years $)$ & 3 & \\
\hline & \multicolumn{2}{|c|}{ Total Adolescent girls } & 9 & \\
\hline \multirow{2}{*}{ FGD2 } & Age Group & $\begin{array}{l}\text { Early adolescent }(14-15 \text { years }) \\
\text { Late adolescent }(16-19 \text { years })\end{array}$ & $\begin{array}{l}2 \\
7\end{array}$ & \multirow{2}{*}{ Chingad Rural Municipality } \\
\hline & \multicolumn{2}{|c|}{ Total Adolescent girls } & 9 & \\
\hline FGD3 & \multicolumn{2}{|c|}{ Total mothers } & 9 & \multirow{2}{*}{$\begin{array}{l}\text { Birendranagar Municipality, Surkhet } \\
\text { Chingad Rural Municipality, Surkhet }\end{array}$} \\
\hline FGD4 & Total mothe & & 9 & \\
\hline
\end{tabular}

\subsection{Knowledge About Reproductive Health Problems}

Majority of adolescent girls have inadequate knowledge about the reproductive health problems. Adolescent girls from the urban area have more knowledge than the rural area. Although they could not define reproductive health problem accurately, most of them were familiar with at least one problem.

"Reproductive health problems mean any problem occurring in female reproduction system like lower abdominal pain during menstruation, excessive bleeding, uterine cancer, backache, lower abdominal pain, uterine prolapse, vaginal discharge, white discharge" (Urban adolescent girl)

During discussion some participants reported that headache and vomiting also come under reproductive health problems. The major source of knowledge was mothers and sisters, while the other sources included school, community, newspapers, neighbors, and non-governmental Organizations. "Madams from NGOs come to teach us about reproductive health. They gather teenagers in one place in school and share the information with us. Sometimes, they also show documentaries related to menstrual hygiene" (Rural adolescent girl)

\subsection{Menstrual Problems Among the Adolescent Girls and Associated Reasons}

"Irregular menstruation, light bleeding, and uterus problem are common in our area. Backache, abdominal pain, itching in the genital area, lower abdominal pain and heavy bleeding are the other problems from which the adolescent girls suffer more" (Rural adolescent girl)

Although similar types of menstrual problems were reported from the urban area, irregular menstruation, itching in the genital area and uterus problem were the most highlighted ones in the rural area. "Poor menstrual hygiene is one of the reasons of menstrual problems" (Urban adolescent girl) "Most of the girls did not share anything about their menstrual problems so they suffered from them" (Rural adolescent girl)

Heavy workload during menstruation, low menstrual hygiene practice, poor nutrition and restriction to certain foods, like cow milk, papaya, etc are the reasons for the occurrence of reproductive and menstrual health problems. "Adolescent girls are not allowed to touch water source during their periods, which result in limited access to water for maintaining their hygiene "(Rural adolescent girl) "To stop this, we should teach them about reproductive health from class 9.... class 6-7 and they should be taught continuously. If we ignore our reproductive health, we females may suffer from different kinds of reproductive diseases, which may lead to death." (Urban adolescent girl)

\subsection{Experience at the Time of Menarche}

While asked about their experience at the time of menarche, most of the girls said they felt shy and anxious. Some of them could not even share it with their mothers. As similar question was asked with mothers as well, where most of them said they were completely unaware about the menstruation phenomenon. They reasoned that no one shared about the process with them and they only heard that females menstruated in their lifetime.

"I saw blood coming from vagina, when $i$ was in school. Then, when I came home and asked with my sister about it .... She said that when we menstruate we are not allowed seeing the sun and faced the boys. Then after, my mother came and told me to go inside the "bhakaari" and covered its top with "supo". I was just 11 and innocent, so I followed her obediently. I stayed there the whole day in the evening my sister came and took me to ours neighbor home and I was hidden there for 11 days. They used to bring me food there. I used to be alone no one was allowed to meet me during those days. (Mother participant urban area)

After 11 days, they bring me out of that house and allowed to go out. I lied to my friends and told them that I went to my mamaghar during those 11 days so not able to come to school. After 22 days there was a small "puja", where they offered me "tika" and new pair of clothes as a gift after that I was allowed to do touch water and do others work.(Mother participant from urban area)

"I was told that there will be a kind of blast- like sound just before menstruation. I was 12 when I first bleed but I did not know about it. I was wearing green surwal and blue frock and it was all wet with blood; I didn't know what is happening to me that day. I talked about it with my aunt and then she confirmed that I was menstruating. She advised me to go to my sister's house because I was not allowed to see my brother and my father. I refused initially but later she convinced me by saying that it might lead to 
my brother's death if I see him at the time of menarche" (Dalit mother participant from urban area)

In the Mid and Far western parts of Nepal, traditional and cultural practices related to menstruation are still followed by women there. There have been in some changes in those practices, yet, some still follow "chaaupadi partha" and the majority of them are restricted to do some specific activities and consume certain food items during menstruation.

"I felt really shy (hehe...). Felt shy........ could not tell about it to others. I was amazed by what was happening to me. I was at school when I bled for the first time and while going home, (Hehehe.....) my pants got stained. When I reached home I was shy to tell my mother about it at first but later after getting changed I told her. Then, she hid me somewhere far. I was told not to see the sun and also my father and brother and like that it happened."(Urban adolescent girl)

"I was shivering excessively later I saw blood and I was taken aback. I asked my sister about it. She told me I was menstruating and advised me not to tell anyone because it was a private matter. She told this is a monthly thing" (Rural adolescent girl)

Even though Surkhet District was declared as "Chhaupadi pratha" free district, people still practice "lukaune" (hidden practice at the time of menarche). However, the duration of "lukaune" has decreased from 11 days to 5 or 3 days but the practice is still followed.

\subsection{Practice and Beliefs Regarding Menstruation}

There is a practice of remaining outdoors for 5 to 7 days of their menstrual cycle each month. This is because of fear of bringing Goddess's curse on their home and family by not touching a man, a cow, water sources and any fruit trees and vegetables in the kitchen garden. If they do, they believed that the tree would stop producing fruit, the cow would stop producing milk and the man would fall ill. Prohibited from bathing during their cycle, women are forced to live in the unsanitary condition of a hut, especially made for them. These are the most common cultural practices/misconception which is still prevalent in Western Nepal.

"In some villages, people still don't give milk to strangers due to fear of someone menstruating touching that milk. They said if menstruating girls drank that milk; the cows/buffaloes would not give milk. Hehe..." (Urban adolescent girl))

"I was kept in a house which was made only for sleeping during menstruation and pregnancy. I had to sleep on the floor. I stayed there for 11 days with my sister in law. She used to serve me food. I was allowed to go to toilet only in the night. Luckily I did not need to go toilet at the day time." (Mother participant from rural area)

"The grass cut by menstruating girl wasn't given to animals". (Mother participant from rural area)

"We were not allowed to go in sunlight. We used to wash our cotton pads at night time and dried inside the room where we stayed. We used to light fire inside the room to dry them faster." (Mother participant urban area)

"Among Brahmin and Chettri: if menstruating girl enters worship room or temple, God will punish them and they will suffer from many problems in their lives. Their family members will be sick. Some say that God will harm the menstruating girls even if they enter the main gate of their homes. If they touch their brothers, they will be cursed. If the plants are touched, they will not give fruits. The flowers and seeds will drop or they will be diseased. They should not touch the papaya tree, baar and peepals tree. There are also.

\subsection{Health Seeking Practice for Menstrual Problem}

Health seeking practice among the adolescence was found poor due to lack of adequate knowledge about the importance of health seeking for the menstrual problem, fear of confidentiality, distance of health facility, user friendly services, and stigma and taboos. Yet, the practice of visiting "dhami jhakri" and then only going to the health facility after the problem has become severe, is still prevalent.

"During menstruation, I had so much pain in my lower abdomen. I used to stand up because I couldn't sit. Then, I visited a "dhami" and he told me "Boksy lageko". When the same thing happened again, my mother told me to go to a hospital but I did not feel comfortable going there because there was a male doctor". (Rural adolescent girl)

"I had pain in my abdomen, excessive headache, lack of interest in doing any work, and also sometimes, I got chills. I had brought medicines from the pharmacies, but they did not improve my condition". (Urban adolescent girl)

Table 2. Factors influencing health seeking behavior.

\begin{tabular}{lll}
\hline Factors & Facilitators & Barriers \\
\hline & & 1) Poor knowledge the regarding menstrual problem \\
& 2) Economic status of family \\
Individual & 3) Interpretation of problem \\
& & 4) Fear of confidentiality \\
& 5) Time taken to make decision \\
& Support from family & 1) Distance of health facility \\
& & 2) Traditional culture \\
Social/Environmental & 3) Stigma/taboos \\
& 4) Low priority to menstrual problems \\
& 5) Decision making power
\end{tabular}




\subsection{Menstruation Practices}

Frequent change of pads, proper management of used pads, frequent bathing and washing of genital organs are included in good menstrual hygiene practices. "We wash cloth pads in the night and dry them inside our rooms, where nobody sees them. Since we are not allowed to dry them in the sunlight, we used to light fire inside our room to dry them faster." (Mother participant rural area)

"We didn't know how to use the pads and how frequently we needed to change them. Yet, now, our daughters teach us to change them every 4 hours". (Mother participant urban area) "I didn't know how frequently it needed to be changed... so, I used to change them when I think it should be changed." (Rural adolescent girl)

"There is one case in our neighborhood. She had some problems in her uterus. I heard it was probably due to lack of sanitation during menstruation, given that she is uneducated. Nobody advised her, so she suffered. But now she is fine after getting proper treatment." (Urban adolescent girl)

There are many similarities and differences in the menstrual practices among mothers and adolescent girls:

1) In older time mothers did not talk about menstruation with their mothers but now they talked about it with their daughters.

2) Before, "Chaau Goth" was used as a place for girls to stay over during their menstruation, but now it used lesser in comparison. However, hiding practice during menarche still not eliminated.

3) Before, there was complete restriction to consume cow milk, papaya and other food items, but now these are allowed to some extent.

4) Nowadays, the use of sanitary pad is common, but before, only clothes could be used.

5) In the past, the females were not allowed to meet the male members of the family nor go in sun light during menstruation, however, now, this is not allowed only in the Brahmin community.

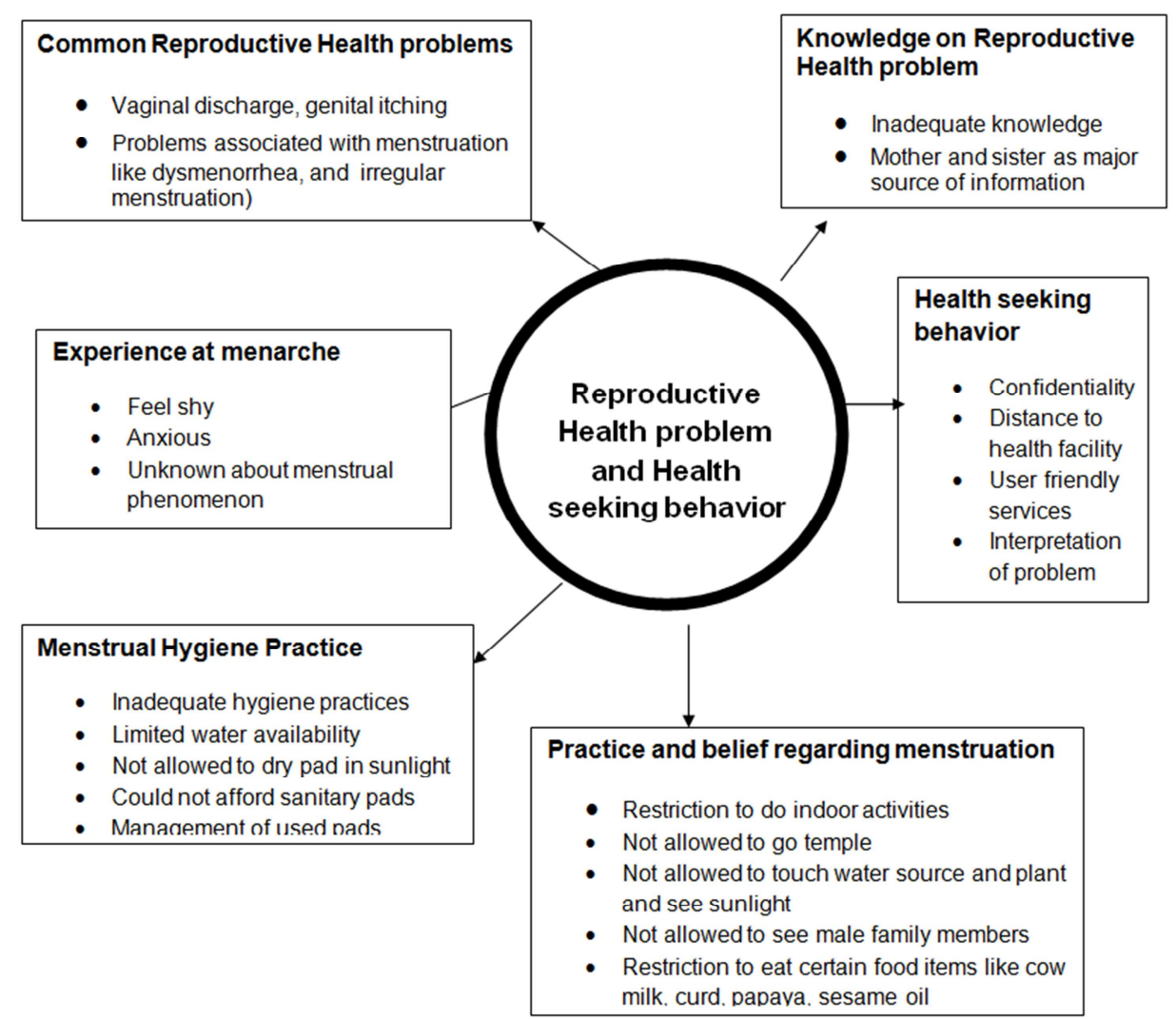

Figure 1. Info-graphs of reproductive health problems and health seeking behavior.

\section{Discussion}

The onset of menstruation is one of the most important changes occurring among the girls during the adolescent years. Although the menstruation is a natural process, it is linked with several misconceptions and practices, which sometimes result into adverse health outcomes. In far and mid-western region of Nepal "Chhaupadi pratha" is highly prevalent. In this region, menstruation is taken as a matter of shame and is not discussed openly, even with mothers and sisters. Our study shows that most of the adolescent girls said they were feeling shy and full of fear. Some of them could not tell with their mother too. Due to inadequate information on the menstruation as a natural phenomenon, many girls get frightened by menstrual flow and are unable to manage it in a safe manner which may lead to reproductive tract infections 
and other reproductive health problems. Similarly, a qualitative study carried out in rural Kenya [8]." and Jordan [9]." showed fear, distraction, confusion and shame were the feelings of girls associated with menstruation at menarche. The reason for fear, tension, shame and helpless may be due to inadequate knowledge, wrong knowledge and misconception in this study.

The similar question was asked with mothers too and most of them said they during their time they were completely unaware about the menstruation phenomenon because no one share with them about this process only they heard female became menstruate during her lifecycle. In province 6 and 7 of our country there was so much painful tradition and culture related to menstruation which is still followed by women who stay there. With the flow of time bring some changes but still some people follow "chaaupadi partha" and majority of them restrict to do some activities and consume some food items during menstruation.

Irregular menstruation, light bleeding, and uterus problem are common menstrual problems. Backache, abdominal pain, itching in genital area, lower abdominal pain and heavy bleeding are the other problems from which the adolescent girls suffer. Health seeking practice among the adolescence for those problems were found poor in this study due to lack of adequate knowledge about the importance of health seeking for menstrual problem, fear of confidentiality, distance of health facility, user friendly services, and stigma and taboos. The practice of visiting "dhami jhakri" and then only going to the health facility after the problem has become severe, is still prevalent. This finding support the study done in Chitradurga where majority $(92.38 \%)$ of girls reported 'Didn't think it was necessary' as a reason for not seeking health care [10]." Similar study conducted in Dhaka shows $41 \%$ mentioned 'no need of treatment' followed by 'lack of knowledge, economic hardship, and shyness to expose to doctor and inadequate service facilities such as "no female doctor available in the hospital [11]." Similarly qualitative study done in Sri Lanka showed the similar reasons for not seeking health services [12]."

Menstrual hygiene practice includes frequently change pads, proper management of used pads, frequent bathing and washing of genital organs. Whereas the study shows some adolescents still did not follow proper hygiene practices. "We wash cloth pads in the night and dry them inside our rooms, where nobody sees them. Since we are not allowed to dry them in the sunlight, we used to light fire inside our room to dry them faster." (Mother participant from rural area). Regarding places to sleep during menstruation, this study found some of them slept inside their home but in a different place, and some slept in a separate house, which was especially made for sleeping during menstruation. Mother participant of FGD said "I was kept in a house which was made only for sleeping during menstruation and pregnancy. I had to sleep on the floor. I stayed there for 11 days. I was allowed to go to toilet only in the night. Luckily I did not need to go toilet at the day time." This finding further supports the result of the previous study conducted in kailali

\section{district [13]."}

In our society due to traditional thoughts there are restrictions to do some activities during menstruation. The result of the present study shows respondents were not allowed attending religious function during menstruation, touch anything in the kitchen, sleep in the same place as earlier, touch father, brother, pregnant women, and newborn child, touch water source and plants and were not allowed to eat certain foods. Which support the findings observed in the study conducted by Shanbhag Det al. where 94.2\% were restricted to go to the place of worship and special functions, $42.6 \%$ avoided specific food items and followed by others [14]." Similar results were found in study done in Raichur in which $100 \%$ respondents were restricted to attend religious functions followed by touching or cooking food $(8.8 \%)$, Sitting or sleeping within house $(1.3 \%)$, going out of the House (1.3\%) and related to diet (1.3\%) [15]." Some previous qualitative study conducted in Nepal $[13,16] . "$ and in study done in Ranchi [17]." also shows similar findings. Restrictions, as produced by myths, misconceptions, superstitions and taboos, is believed to have the potential to spoil food, plant, biological and social processes and even the health of their brothers if they are touched during the phase of menstruation shown in a study in Nepal $[13,18]$."

Remain outdoors for 5 to 7 days of their menstrual cycle in each month because of fear of bringing Goddess's curse on their home and family by not touching a man, a cow, water sources and any fruit trees and vegetables in the kitchen garden. If they do, they believed that the tree would stop producing fruit, the cow would stop producing milk and the man would fall ill. Prohibited from bathing during their cycle, women are forced to live in the unsanitary condition of a hut, especially made for them. Even they prefer to manage menstrual problems at their home by taking rest, oil massage, drink hot water and soup and self-medication rather than visiting health facility. These are the most common cultural practices/misconception which is still prevalent in Western Nepal. Previous study done in Nepal [19, 21]." and Delhi [20]." supported this finding.

\section{Conclusion}

This study shows that most of the adolescent girls were shy and anxious during menarche and did not communicate properly with their parents regarding menstrual problems. Although menstruation is normal physiology, in our society menstruating woman is categorized as "impure", "polluted" and victimized in the form of "Chhaupadi Pratha". The misconception that "females touching others during their menstruation brings God's curse to their home and families" is still rampant in Western Nepal. Concerned authorities should conduct health promotion activities to address proper menstrual hygiene and practices among adolescent girls in western Nepal. The findings of this study will be useful for healthcare professional and administrator to improve the reproductive health status of the adolescent. 


\section{Conflict of Interest}

All the authors do not have any possible conflicts of interest.

\section{Author's Contribution}

$\mathrm{KB}, \mathrm{RK}, \mathrm{AG}$ and PP made substantial contributions to the conception or design of the work. KB, PPS, TBA and RS were involved in the acquisition of data (conduct FGD), cleaned, analyzed and interpreted the data. KB, RK, AG and PP revised the work critically. RK, AG and PP gave final approval of the version to be published. All agreed to be accountable for all aspects of the work in ensuring that questions related to the accuracy or integrity of any part of the work are appropriately investigated and resolved. All authors read and approved the final manuscript.

\section{Funding}

No any funding source for conducting the study.

\section{Acknowledgements}

I would like to convey my sincere gratitude to School of Public Health and Community Medicine and all the faculties for providing me this opportunity I am very grateful for the guidance of Chief guide Dr. Rajendra Karkee, Associate Professor, School of Public Health and Community Medicine, co-guides Prof. Dr. Anup Ghimire, Chief, School of Public Health and Community Medicine and Dr. Prajjwal Pyakurel, School of Public Health and Community Medicine. I am extremely thankful to principals, teachers, mothers and students of all the sampled secondary level public schools of Surkhet District for their timely support, cooperation and honest information during data collection.

\section{References}

[1] Nepal Government. Nepal Population Report 2016.

[2] Kulkarni MV, Durge P. Reproductive health morbidities among adolescent girls: Breaking the silence. Ethno Med. 2011; 5 (3): 165-8.

[3] World Health Organization. Adolescent development. Available from: http://www.who.int/maternal_child_adolescent/topics/adolesc ence/development/en/.

[4] Anoop Khanna. Menstrual Practices and Reproductive Problems: A Study of Adolescent Girls in Rajasthan. Journal of Health Management. 2005.

[5] Joshi LR. Chhaupadi Partha: Socio-cultural Violence against Women in the Far Western Region of Nepal. Journal of Nepal Public Health Association. 2015; 6 (1).

[6] Trudy D'souza. Gyanetone Official Blog. October 29, 2015
[7] FieldBulletin "ChaupadiIn The Far-West". 2011 (1). Available from: http://www.ohchr.org/Documents/Issues/Water/ContributionsS tigma/oth ers/field_bulletin.

[8] McMahon SA, Winch PJ, Caruso BA, Obure AF, Ogutu EA, Ochari IA, et al. 'The girl with her period is the one to hang her head'Reflections on menstrual management among schoolgirls in rural Kenya. BMC international health and human rights. 2011; 11 (1): 7.

[9] Al Omari O, Razeq NMA, Fooladi MM. Experience of menarche among Jordanian adolescent girls: An interpretive phenomenological analysis. Journal of pediatric and adolescent gynecology. 2016; 29 (3): 246-51.

[10] Ramya V, Reddy MR, Sridevi B. A Study on Reproductive Morbidity and Menstrual Hygiene among Adolescent Girls of Urban Slum Area of Chitradurga. National Journal of Community Medicine. 2016; 7 (3): 180-3.

[11] Rahman MM, Kabir M, Shahidullah M. Adolescent selfreported reproductive morbidity and health care seeking behaviour. Religion. 2004; 33 (6): 1248.

[12] Pokhrel R. Adolescents not getting friendly health services The Himalayan Times. July 11, 2015.

[13] Hamal M, Susma K. Hygiene, Health Problems and Sociocultural practices: what school girls do during menstruation age. 2014; 12 (13): 14.

[14] Shanbhag D, Shilpa R, D'souza N, Josephine P, Singh J, Goud B. Perceptions regarding menstruation and practices during menstrual cycles among high school going adolescent girls in resource limited settings around Bangalore city, Karnataka, India. International Journal of Collaborative Research on Internal Medicine \& Public Health. 2012; 4 (7): 1353-62.

[15] Ade A, Patil R. Menstrual hygiene and practices of rural adolescent girls of Raichur. International Journel of Bio Med Research. 2013; 4 (2): 3014-7.

[16] Khanal P. Adolescents Knowledge and Perception of Sexual and Reproductive Health and Services-A Study from Nepal. 2016.

[17] Kumar A, Srivastava K. Cultural and social practices regarding menstruation among adolescent girls. Social work in public health. 2011; 26 (6): 594-604.

[18] Pradhan WA. Is menstrual hygiene and management an issue for adolescent school girls? A comparative study of four schools in different settings of Nepal. 2009.

[19] GC Sona, Koirala Priti. Menstruation among Nepalese Adolescent girls. 2013.

[20] Garg S, Sharma N, Sahay R. Socio-cultural aspects of menstruation in an urban slum in Delhi, India. Reproductive health matters. 2001; 9 (17): 16-25.

[21] S. B. Parajuli et al. Chaupadi during menstruation still a major community health challenge: perspective from Mid-Western Nepal 2018 